\title{
Concordancia entre las técnicas de hemaglutinación indirecta e inmunoabsorción ligado a enzimas en el diagnóstico de toxoplasmosis porcina
}

\author{
Concordance between indirect hemagglutination and enzyme-linked \\ immunosorbent techniques in the diagnosis of porcine toxoplasmosis \\ Marlene Gonzales L. ${ }^{1}$, Christian Luyo A. ${ }^{1}$, Rosa Pinedo V. ${ }^{1}$, Amanda Chávez V. ${ }^{1}$, \\ Eva Casas A. ${ }^{1,2}$
}

\section{Resumen}

El objetivo del presente estudio fue determinar la concordancia entre las pruebas de hemaglutinación indirecta (HAI) y el ensayo de inmunoabsorción ligado a enzimas (ELISA) para detectar IgG anti-Toxoplasma gondii en el diagnóstico de toxoplasmosis porcina. Se colectaron muestras de sangre de 407 cerdos en la fase de acabado, provenientes de crianzas porcinas ubicadas en la franja costera del departamento de Lima, Perú. La concordancia de las técnicas diagnósticas y la seroprevalencia correspondiente se evaluaron mediante el índice de Kappa y la prueba de McNemar. Los resultados indicaron que la concordancia entre las pruebas de HAI y ELISA a través del índice de Kappa fue de $26 \%$, considerándose de tipo regular, con valores de $18.7 \pm 3.8 \%$ por el método de HAI y $14.7 \pm 3.4 \%$ por el método de ELISA; mientras que no se encontró diferencias significativas mediante la prueba de McNemar, lo cual podría sugerir que ambas técnicas son mutuamente reemplazables. No obstante, se concluye que, como la correlación es regular, no se recomienda su reemplazo.

Palabras clave: porcinos; Toxoplasma gondii; ELISA indirecta; HAI

\section{Abstract}

The aim of the present study was to determine the concordance between the indirect hemagglutination test (HAI) and the enzyme-linked immunosorbent assay (ELISA) to detect anti-Toxoplasma gondii $\mathrm{IgG}$ in the diagnosis of porcine toxoplasmosis. Blood samples were collected from 407 pigs in the finishing phase, from pig farms located in the

\footnotetext{
${ }^{1}$ Laboratorio de Microbiología y Parasitología Veterinaria, Facultad de Medicina Veterinaria, Universidad Nacional Mayor de San Marcos, Lima, Perú

${ }^{2}$ E-mail: evacasas99@gmail.com
}

Recibido: 13 de marzo de 2018

Aceptado para publicación: 8 de noviembre de 2018 
coastal strip of the department of Lima, Peru. The concordance of the diagnostic techniques and the corresponding seroprevalence were evaluated using the Kappa index and the McNemar test. The results indicated that the concordance between the HAI and ELISA tests through the Kappa index was $26 \%$, considering the regular type, with values of $18.7 \pm 3.8 \%$ by the HAI method and $14.7 \pm 3.4 \%$ by the method of ELISA; while no significant differences were found by the McNemar test, which could suggest that both techniques are mutually replaceable. However, it is concluded that, since the correlation is regular, it is not recommended its replacement.

Key words: swine; Toxoplasma gondii; indirect ELISA; HAI

\section{INTRODUCCIÓN}

Toxoplasma gondii es un protozoario intracelular obligado de distribución mundial que tiene a los felinos domésticos y silvestres como hospedadores definitivos y a una amplia gama de vertebrados como hospedadores intermediarios. La ingestión de carne cruda o mal cocida, infectada con quistes tisulares, o el consumo de alimentos y agua contaminados con ooquistes esporulados son las rutas más comunes de infección (López et al., 2005; Dubey y Jones, 2008).

La infección por $T$. gondii tiene manifestaciones clínicas diversas e inespecíficas, lo que hace difícil precisar el diagnóstico, por lo que se debe tener cuidado con el diagnóstico diferencial (Dubey, 2010). Como en muchas enfermedades infecciosas, sobre todo en aquellas que son clínicamente inaparentes, el diagnóstico se basa en pruebas indirectas como la serología, que consiste en la determinación de anticuerpos específicos contra el parásito (Bastien, 2002). Entre las pruebas serológicas disponibles para el diagnóstico de toxoplasmosis se tiene la Hemaglutinación Indirecta (HAI), el Ensayo de Inmunoabsorción Ligado a Enzimas (ELISA) y la Inmunofluorescencia Indirecta (IFI) (Cortes y Mancera, 2009), la técnica de aglutinación modificada (MAT), considerada como referencia para la validación de otras pruebas serológicas (Klun et al., 2006; Sroka et al., 2011), y la técnica de Sabin-Feldman o Dye
Test, conocida como prueba confirmatoria (Joynson y Wreghitt, 2001). Sin embargo, la prueba de Hemaglutinación Indirecta (HAI) es la que se utiliza para el diagnóstico de rutina en clínicas y hospitales veterinarios (Cerro et al., 2009), por ofrecer resultados rápidos y una mayor facilidad de ejecución e interpretación de resultados (Suárez et al., 2002).

Por otro lado, el ELISA ha mostrado claras ventajas sobre otras pruebas, dado que se pueden analizar muchas muestras en forma simultánea con equipos de fácil uso, comparado con la prueba de inmunofluorescencia, y los resultados obtenidos ofrecen mayor confiabilidad que las obtenidas con la hemaglutinación (Dubey, 2010). Además, posee alta sensibilidad y especificidad detectando tanto infecciones recientes como latentes (Steinparzer et al., 2014). Es así que el objetivo del presente estudio fue determinar la concordancia entre las pruebas de HAI y el ELISA para el diagnóstico de Toxoplasma gondii en cerdos.

\section{Materiales y Métodos}

El estudio se realizó en siete granjas porcinas tecnificadas y en 10 granjas no tecnificadas localizadas en los distritos de Huacho, Huaral, Ventanilla, Villa E1 Salvador, Lurín y Chilca, todos ellos en la franja costera del departamento de Lima, Perú, en- 
tre los meses de enero y marzo de 2014 y 2015. Se realizó el seguimiento de los animales en los camales de beneficio para la posterior toma de muestra de sangre, previo consentimiento informado. El procesamiento de las muestras se llevó a cabo en el Laboratorio de Parasitología de la Facultad de Medicina Veterinaria de la Universidad Nacional Mayor de San Marcos, distrito de San Borja, Lima.

El tamaño muestral se determinó mediante la fórmula de proporción de poblaciones finitas con $95 \%$ de confianza y $5 \%$ de precisión (Daniel, 1996). Se utilizó en el cálculo el tamaño de la población porcina de Lima (388 793 animales) (INEI, 2013) y $32.3 \%$ como prevalencia referencial (SuárezAranda et al., 2000), lo que dio como resultado un mínimo de 407 muestras. Para el efecto del estudio, se trabajó con 264 cerdos de cinco meses de granjas tecnificadas y con 143 cerdos $\geq 7$ meses de criaderos no tecnificados, en ambos casos sin distinción de sexo y en etapa de acabado.

Los productores firmaron el Consentimiento Informado previo a la toma de las muestras. Estas fueron tomadas al momento del sacrificio, durante el proceso de degüello, en tubos de boca ancha sin anticoagulante. Se tomó aproximadamente 5-8 $\mathrm{ml}$ de muestra de sangre que fueron llevadas al laboratorio donde se obtuvieron los sueros por centrifugación a $1500 \mathrm{rpm}$ durante $5 \mathrm{~min}$, conservándose en viales a $-20{ }^{\circ} \mathrm{C}$ hasta su procesamiento y análisis serológico.

Se utilizó el «kit» comercial PrioCHECK Porcine Toxoplasma Ab (Prionics Ag, Suiza), siguiendo las instrucciones de acuerdo con el protocolo del fabricante. El kit posee una sensibilidad de $98.9 \%$ y una especificidad de $92.7 \%$ (Basso et al., 2013). La lectura de la absorbancia se realizó en un lector automático (Kayto) de ELISA con filtro de $450 \mathrm{~nm}$. Para la interpretación de los resultados se calculó el porcentaje de positividad (PP\%) y fueron considerados positivos cocientes iguales o mayores a $20 \%$.
Para la técnica de hemaglutinación indirecta (HAI) se utilizó un kit comercial (Toxotest) para uso humano, siguiendo las instrucciones de acuerdo con el protocolo del fabricante (Wienner Lab, 2000). El kit posee una sensibilidad de $91.0 \%$ y una especificidad de $96.4 \%$ en sueros de humanos (Diaz et al., 2001; Dabanch, 2003). Se prepararon diluciones de las muestras de 1:16 a 1:2048, considerándose como positivo títulos iguales o mayores a 1/64, basado en estudios previos (Romero y Sogbe, 2005; Romero et al., 2007). Se siguieron las instrucciones de acuerdo a lo establecido por el fabricante.

Se evaluó el grado de concordancia mediante el índice de Kappa, considerándose la interpretación establecida por Landis y Koch (1977) de 0: pobre; >0-0.2: leve; 0.210.40: regular; 0.41-0.60: moderada; 0.61-0.8: buena; 0.81-1: perfecta. Asimismo, se utilizó la prueba de Mc Nemar para determinar si las pruebas son mutuamente reemplazables.

\section{Resultados}

La seroprevalencia de Toxoplasma gondii en cerdos de Lima mediante las técnicas de HAI y ELISA indirecto fue de $18.7 \pm$ $3.8 \%(76 / 407)$ y de $14.7 \pm 3.4 \%(60 / 407)$, respectivamente.

Cuadro 1. Distribución de sueros según los resultados de las técnicas de ELISA indirecto y hemaglutinación indirecta (HAI) para la detección de Toxoplasma gondii en cerdos de Lima, Perú (2015)

\begin{tabular}{cccc}
\hline \multirow{2}{*}{ HAI } & \multicolumn{2}{c}{ ELISA } & \multirow{2}{*}{ Total } \\
\cline { 2 - 3 } & + & - & \\
\hline+ & 26 & 50 & 76 \\
- & 34 & 297 & 331 \\
\hline Total & 60 & 347 & 407 \\
\hline $\mathrm{K}=26.0 \%$ & & &
\end{tabular}


En el Cuadro 1 se presentan los resultados serológicos de los sueros de cerdos mediante las técnicas de ELISA indirecto y HAI, agrupados de acuerdo con su concordancia. La prueba de Kappa arroja un valor de concordancia regular de 0.26 , mientras que la prueba de McNemar no detectó diferencia significativa entre las técnicas, ya que el coeficiente calculado fue de 3.28, siendo menor que el de tabla, demostrando que una prueba puede ser reemplazada por la otra, pero con una correlación débil, de allí que no se recomienda.

\section{Discusión}

El diagnóstico de infección por $T$. gondii en porcinos se basa usualmente en la detección de anticuerpos específico, habiendo varios kits comerciales (Jiang et al., 2008) con grandes diferencias en sensibilidad y especificidad (Huerta et al., 2006), cuyo uso depende de las facilidades de equipos y reactivos disponibles en el laboratorio.

La técnica de ELISA es una prueba para la detección cualitativa y cuantitativa de anticuerpos IgG contra $T$. gondii (Romero et al., 1995). Se disponen de varios kits comerciales con diferentes antígenos y puntos de corte (Dubey, 2009), así como niveles de sensibilidad (57.3-65.2\%) y especificidad (97.4-99.4\%) (Basso et al., 2013; Steinparzer et al., 2014).

La técnica de HAI emplea glóbulos rojos de carnero sensibilizados (Balfour et al., 1982) con antígenos citoplasmáticos y de superficie del parásito para detectar anticuerpos totales (IgG e IgM), incrementando la sensibilidad de la prueba. Por lo tanto, permite la detección precoz de la infección (Wiener Lab, 2000). La técnica presenta una sensibilidad de $29.4 \%$ y una especificidad de $98.3 \%$ (Dubey et al., 1995) y se viene utilizando en clínicas y hospitales veterinarios (Suárez et al., 2002).
El 26\% de coeficiente de concordancia Kappa fue relativamente similar al 36.6\% obtenido por Suárez et al. (2002) en cerdos, donde consideraron al ELISA como prueba de referencia; sin embargo, en el presente estudio la prueba de McNemar no demostró significancia, pero dado que el índice Kappa presentó una concordancia regular, indica que estas pruebas no son mutuamente reemplazables.

Las técnicas de HAI y ELISA han venido siendo comparadas con muestras de diferentes especies y agentes infecciosos (Figueiredo et al., 2001), observando ventajas de la técnica HAI sobre el ELISA, pues es una prueba simple y confiable, dado que posee la facilidad de evaluar cada muestra de forma independiente con pequeña cantidad de suero (Suárez et al., 2002). Además, permite detectar el título de anticuerpo tomando la mayor dilución en la cual se observa hemaglutinación franca, utilizando punto de corte de 1:16 (Triolo-Mieses y TraviezoValles, 2006) y 1:64 (Zou et al., 2009).

La técnica de ELISA por su parte presenta ventajas respecto al HAI, pues permite un diagnóstico rápido, con disponibilidad de una gran variedad de kits comerciales que determinan IgM e IgG independientemente, el cual es eficaz para realizar monitoreos de rutina en un hato porcino (Handman et al., 1980; Kasper et al., 1983). Por ejemplo, Lind et al. (1997) encontraron IgM de corta duración ( 2 semanas) y posteriormente IgG, que se mantuvo durante 3-4 meses. Para el caso del presente estudio, mediante la técnica utilizada se detectó IgG a las 2 semanas posinfección y un incremento de los niveles de anticuerpos a las 11 semanas posteriores.

La técnica de ELISA enfocada en el diagnóstico de toxoplasmosis porcina varía en cuanto a la sensibilidad y especificidad dependiendo del antígeno utilizado. Así, Gamble et al. (2005), utilizando un ELISA comercial de antígeno P30 reportan una sensibilidad de $85.7-88.6 \%$ y una especificidad de 94.6- 
98.0\%, mientras que Basso et al. (2013), utilizando el mismo tipo de kit del presente estudio determinaron una sensibilidad de $88.9 \%$ y una especificidad de $92.7 \%$.

\section{Conclusiones}

El grado de concordancia en el diagnóstico de toxoplasmosis porcina entre las pruebas de HAI (18.7\%) y ELISA (14.7\%) mostraron valores de $26.0 \%$ para Índice de Kappa y 3.28 para Mc Nemar, demostrándose que ambas pruebas pueden ser reemplazables, pero debido a que la correlación es regular, no se recomienda su reemplazo, por el contrario, ambas pruebas se pueden complementar a fin de obtener un diagnóstico preciso.

\section{Literatura Citada}

1. Balfour AH, Fleck DG, Hugues HP, Sharp D. 1982. Comparative study of three tests (dye test, indirect haemagglutination test, latex agglutination test) for antibodies to Toxoplasma gondii in human sera. J Clin Pathol 35: 228-232.

2. Basso W, Hartnack S, Pardini L, Maksimov P, Koudela B, Venturini MC, Schares G, et al. 2013. Assessment of diagnostic accuracy of a commercial ELISA for the detection of Toxoplasma gondii infection in pigs compared with IFAT, TgSAG1-ELISA and Western blot, using a Bayesian latent class approach. Int J Parasitol 43: 565570. doi: 10.1016/j.ijpara.2013.02.003

3. Bastien P. 2002. Molecular diagnosis of toxoplasmosis. T Roy Soc Trop Med H 96(Suppl 1): 205-215.

4. Cerro L, Chávez A, Casas E, Suárez F, Rubio A. 2009. Frecuencia de Toxoplasma gondii en gatos de Lima Metropolitana y concordancia entre las técnicas de Inmunofluorescencia Indirecta y Hemaglutinación Indirecta. Rev Inv Vet Perú 20 (2): 285-290.
5. Cortes LJ, Mancera L. 2009. Concordancia entre ELISA e IFI para la determinación de anticuerpos tipo IgG contra Toxoplasma gondii. Infectio 13: 76-82. doi: 10.1016/S0123-9392(09)70728-3

6. Dabanch J. 2003. Zoonosis. Rev Chil Infectol 20: 47-51.

7. Daniels W. 1996. Bioestadística: base para el análisis de la ciencia de la salud. $5^{\text {a }}$ ed. México: Limusa. 736 p.

8. Diaz O, Parra A, Araujo M. 2001. Seroepidemiología de la toxoplasmosis en una comunidad marginal del municipio Maracaibo, estado Zulia. Invest Clín 42: 107-121.

9. Dubey JP. 2009. Toxoplasmosis in pigs. The last 20 years. Vet Parasitol 164: 89103. doi: 10.1016/j.vetpar.2009.-05.018.

10. Dubey JP. 2010. Toxoplasmosis of animals and humans. 2a ed. Maryland: CRC Press. 336 p.

11. Dubey JP, Jones JL. 2008. Toxoplasma gondii infection in humans and animals in the United States. Int $\mathbf{J}$ Parasitol 38: 1257-1278. doi: 10.1016/ j.ijpara.2008.03.007

12. Dubey JP, Thulliez P, Weigel RM, Andrews CD, Lind P, Powell EC. 1995. Sensitivity and specificity of various serologic tests for detection of Toxoplasma gondii infection in naturally infected sows. Am J Vet Res 56: 10301036.

13. Figueiredo JF, Silva DA, Cabral DD, Mineo JR. 2001. Seroprevalence of Toxoplasma gondii infection in goats by the indirect haemagglutination, immuno-fluorescence and immunoenzymatic test in the region of Uberlandia Brazil. Mem IOswaldo Cruz 96: 687-692. doi: 10.1590/S007402762001000500019

14. Gamble HR, Dubey JP, Lambillotte DN. 2005. Comparison of a commercial ELISA with the modified agglutination test for detection of Toxoplasma infection in the domestic pig. Vet Parasitol 128: 177-181. doi: 10.1016/ j.vetpar.2004.11.019 
15. Handman E, Goding JW, Remington JS. 1980. Detection and characterization of membrane antigens of Toxoplasma gondii. J Immunol 124: 2578-2583.

16. Huerta O, Chávez A, Casas E, Falcón N, Raymundo F. 2006. Concordancia entre las pruebas de hemaglutinación indirecta e inmunofluorescencia indirecta para determinar la prevalencia de Toxoplasma gondii en ovinos. Rev Inv Vet Perú. 17: 178-183. doi: 10.15381/ rivep. v17i2.1538

17. [INEI] Instituto Nacional de Estadística e Informática. 2013. IV Censo Nacional Agropecuario 2012. [Internet], [30 de agosto de 2014]. Disponible en: https://www.agrorural.gob.pe/ dmdocuments/resultados.pdf

18. Jiang T, Gong D, Ma L, Nie H, Zhou Y, Yao B, Zhao J. 2008. Evaluation of a recombinant MIC3 based latex agglutination test for the rapid serodiagnosis of Toxoplasma gondii infection in swines. Vet Parasitol 158: 5156. doi: 10.1016/j.vetpar.2008.07.03

19. Joynson D, Wreghitt T. 2001. Toxoplasmosis. A comprehensive clinical guide. New York. Cambridge University Press. 409p

20. Kasper LH, Crabb JH, Pfefferkorn ER. 1983. Purification of a major membrane protein of Toxoplasma gondii by immunoabsorption with a monoclonal antibody. J Immunol 130: 2407-2412.

21. Klun I, Djurkovia-Djakoviae O, Katice-Radivojeviae $S$, Nikoliae A. 2006. Cross sectional survey on Toxoplasma gondii infection in cattle, sheep and pigs in Serbia: seroprevalence and risk factors. Vet Parasitol 135: 121131. doi: 10.1016/j.vetpar.2005.08.010

22. Landis JR, Koch GG. 1977. The measurement of observer agreement for categorical data. Biometrics 33: 159-179.

23. Lind P, Haugegaard J, Wingstrand A, Henriksen SA. 1997. The time course of the specific antibody response by various ELISAs in pigs experimentally infected with Toxoplasma gondii. Vet
Parasitol 71: 1-15. doi: 10.1016/S03044017(97)00010-1

24. López C, Díaz J, Gómez J. 2005. Factores de riesgo en mujeres embarazadas, infectadas por Toxoplasma gondii en Armenia-Colombia. Rev Salud Públ 7: 180-190.

25. Romero J, Sogbe E, Diaz C. 2007. Estudio serológico e histopatológico de la infección por Toxoplasma gondii en cerdos del estado Aragua-Venezuela. Rev Fac Cs Vets UCV 48: 85-95.

26. Romero J, Sogbe E. 2005. El cerdo (Sus scrofa), fuente de infección de Toxoplasma gondii al humano en el Estado Aragua, Venezuela. B Malariol Salud Amb 45: 111-117.

27. Romero T, Bermúdez M, Dosil P, Montiel M. 1995. Estudio comparativo entre los métodos de hemaglutinación e inmunoanálisis enzimático en el diagnóstico de la toxoplasmosis. Kasmera 23: 69-88.

28. Sroka J, Karamon J, Cencek T, Dutkiewicz J. 2011. Preliminary assessment of usefulness of cELISA test for screening pig and cattle populations for presence of antibodies against Toxoplasma gondii. Ann Agr Env Med 18: 335-339.

29. Steinparzer R, Reisp K, Grünberger B, Köfer J, Schmoll F, Sattler T. 2014. Comparison of different commercial serological tests for the detection of Toxoplasma gondii antibodies in serum of naturally exposed pigs. Zoonoses Public Hlth 62: 119-124. doi: 10.1111/ zph.12122

30. Suárez F, Andrade H, Galisteo A, Miguel O. 2002. Concordancia de las pruebas de ELISA y hemaglutinación indirecta en el diagnóstico de la toxoplasmaosis porcina. Rev Inv Vet Perú 13: 84-86. doi: 10.15381/rivep.v13i1.1712

31. Suárez-Aranda F, Galisteo AJ, Hiramoto RM, Cardoso RP, Meireles LR, Miguel O, Andrade HF. 2000. The prevalence and avidity of Toxoplasma gondii $\operatorname{IgG}$ antibodies in pigs from Brazil and Peru. Vet Parasitol 91: 23-32. 
32. Triolo-Mieses M, Traviezo-Valles $L$. 2006. Seroprevalencia de anticuerpos contra Toxoplasma gondii en gestantes del municipio Palavecino, Estado Lara, Venezuela. Kasmera 34: 7-13.

33. Waltman WD, Dreesen DW, Prickett MD, Blue JL, Oliver DC. 1984. Enzyme-linked immunosorbent assay for the detection of toxoplasmosis in swine: Interpreting assay results and comparing with other serologic tests. Am J Vet Res 45: 1719-1725.

34. Wiener Lab. 2000. Toxotest HAI. Prueba de hemoaglutinación indirecta. Buenos Aires, Argentina: Winner Group Lab. 8 p.

35. Zou FC, Sun XT, Xie YJ, Li B, Zhao GH, Duan G, Zhu XQ. 2009. Seroprevalence of Toxoplasma gondii in pigs in Southwestern China. Parasitol Int 58: 306-307. doi: 10.1016/ j.parint.2009.-06.002 